\title{
A NOVEL APPROACH FOR EFFICIENT SKULL STRIPPING USING MORPHOLOGICAL RECONSTRUCTION AND THRESHOLDING TECHNIQUES
}

\author{
M.Ramesh ${ }^{1}$, P.Priya ${ }^{2}$, Punal.M.Arabi ${ }^{3}$ \\ ${ }^{1}$ Assistant Professor/ECE, Vivekanandha Institute of Engineering and Technology for Women, Tamil Nadu, India, \\ ${ }^{2}$ Assistant Professor/ECE, Vivekanandha Institute of Engineering and Technology for Women, Tamil Nadu, India, \\ ${ }^{3}$ Head of the Department/ECE, Vivekanandha Institute of Engineering and Technology for Women, Tamil Nadu, India,
}

\begin{abstract}
Brain is the part of the central nervous system located in skull. For the diagnosis of human brain bearing tumour, skull stripping plays an important pre-processing role. Skull stripping is the process separating brain and non-brain tissues of the head which is the critical processing step in the analysis of neuroimaging data. Though various algorithms have been proposed to address this problem, challenges remain. In this paper a new efficient skull stripping method for magnetic resonance images (MRI) is proposed. This method adopts a two-step approach; in the first step an improved systematic application of morphological reconstructions operations is done for the brain image and in the second step, a thresholding based technique is used to extract the brain inside the skull. This paper experimented on Axial PD and FLAIR MRI brain images.
\end{abstract}

Index Terms: Skull stripping, thresholding, morphological reconstruction, Axial PD and FLAIR MRI images of brain.

$* * *$

\section{INTRODUCTION}

Skull -stripping is the process of segmenting brain from non-brain tissues (e.g skull, scalp, eyes, and neck) in wholehead magnetic resonance (MRI) images. Skull stripping is one of the pre-processing phases in image processing of brain for detection of brain tumor. Skull stripping, an important task in neuroimage study is a difficult task due to various problems like shape of brain, intensity of the MR images and similarity of intensity values in brain and non brain tissues. The brain portion must be extracted from the skull before the application of segmentation, classification and registration processing algorithms. Neha Tirpude et.al [1] provide a survey of various image segmentation methods that have been applied to brain MRI images.

Studies of brain segmentation and analysis that have employed mathematical morphology are discussed by many authors [2][3][4][5].Gonzales and woods[6] defined mathematical morphology as a tool for extracting useful image components such as skeletons, boundaries etc. Erosion and dilation are two basic operators in the area of mathematical morphology. Sajjad Mohsin et.al [7] employed mathematical morphological algorithms for the removal of skull, after the detection of false background. This method fails on low contrast and noisy images.

K.Somasundaram et.al [8] proposed combination of $\mathrm{k}$ means algorithm and curve fitting using quadratic equation for brain extraction. Orazio Gambino et.al [9] proposed a method for brain extraction by making use of fuzzy c-means segmentation [10] and morphological operators. These methods avoid the processing of back ground and skull areas. But unable to handle noisy data, randomly choosing the cluster centre it cannot lead to the fruitful result.

Thresholding is one of the oldest techniques in image segmentation technique. In this method the image is said to be composed of different gray scale ranges. The threshold value groups the pixels into such a manner that intensity less than the threshold value is grouped as one class and the intensity of the pixel greater than the threshold value is grouped as a another class. A binary image can be obtained when thresholding a gray scale image. Rosniza Roslan [11] showed that thresholding the brain could be used for skull stripping.

Region growing [12][13] is a region based image segmentation technique, also called a pixel based image segmentation method because it involves the selection of initial seed points. This approach is to segmenting neighbouring pixels of initial seed points and determines whether the pixel neighbours should be added to the region. Region growing techniques [14] can be affected due to noise resulting in presence of holes in the image or may lead to disconnected regions. The disadvantage of this algorithm is that user has to select the seed regions and threshold values. Thus J.G.Park et al [15] addressed this problem by 
introducing a 2D region growing algorithm that automatically selects seed regions corresponds to the brain and non-brain regions.F.Segonne et.al [16] proposes a hybrid approach that uses for skull stripping.

The watershed algorithm [17][18] is intensity based approach that operates under the assumption of white matter connectivity and segments the image into brain and nonbrain components. But it produces over segmentation problem. A.H.Zhuang et.al [19] developed an automatic skull stripping model to remove the skull in MRI brain images. K.Somasundram et.al [20] presents a comparative survey on segmentation techniques used for MR Brain Images.

\section{PROPOSED METHOD}

The proposed method is a two stage process. The first process uses morphological reconstruction to produce a primary segmentation i.e mask of the input image, while the second process applies thresholding to the primary segmentation to obtain the final skull stripped image. The flowchart of the proposed method is in Figure 2.

Fig -1 shows two sample images taken on which the proposed method is experimented.

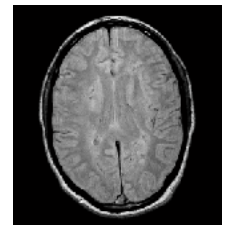

(a)

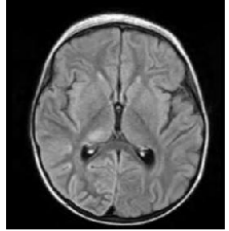

(b)
Fig -1: (a) and (b) MRI Brain Images(Input images)

\subsection{Erosion}

The erosion operator takes two pieces of data as inputs. The first is the image which is to be eroded. The second is a set of coordinate points known as structuring element, which determines the precise effect of the erosion on the input image. Erosion is the counter-process of dilation. If dilation enlarges image then erosion shrinks the image. The way the image is shrunk is determined by the structuring element. The structuring element is normally smaller than the image with a $3 \times 3$ size.

This will ensure faster computation time when compared to larger structuring-element size. Almost similar to the dilation process, the erosion process will move the structuring element from the left to right and top to bottom. At the centre position, indicated by the centre of the structuring element, the process will look for whether there is a complete overlap with the structuring element or not. If there is no complete overlapping then the centre pixel indicated by the centre of the structuring element will be set white or 0 .

Let us define $\mathrm{A}$ as the reference input image and $\mathrm{X}$ as the structuring element. Eroded image is defined B

$$
B=A \ominus X=\left\{Z \mid(\mathrm{X})_{\mathrm{Z}} \sqsubseteq \mathrm{A}\right\}
$$

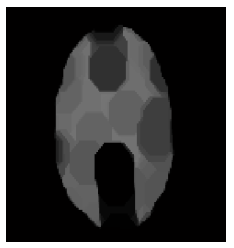

(a)

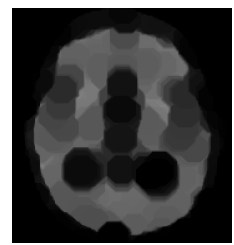

(b)
Fig -2 : (a) and (b) Eroded images

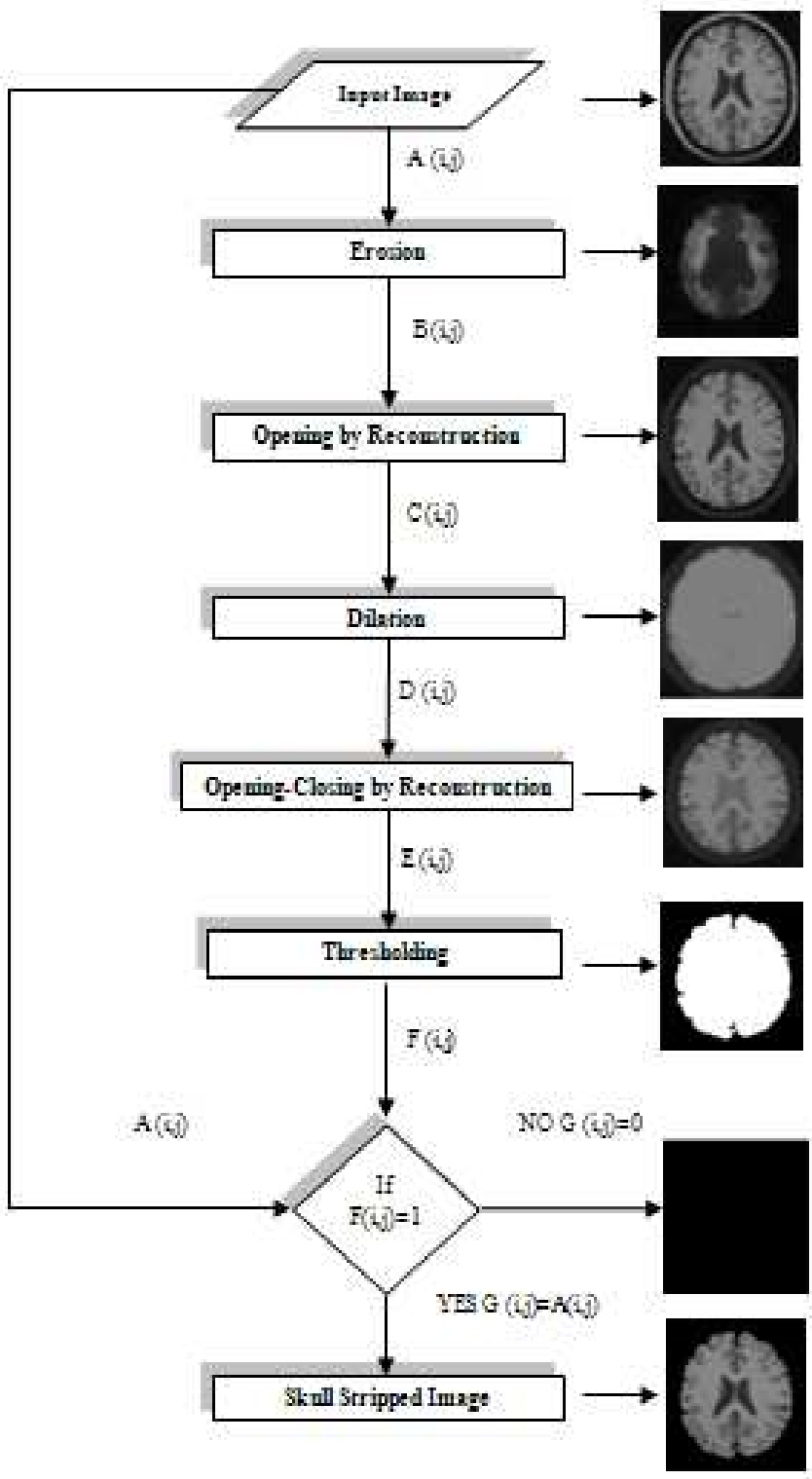

Fig-3: Flowchart of proposed method

\subsection{Opening by Reconstruction}

The opening by Reconstruction restores the original shapes of the objects that remain after erosion. Performing morphological reconstruction using the eroded image as the marker and the original image as the mask is termed as opening by reconstruction. The opening by reconstruction of an image $\mathrm{B}$ using structuring element $\mathrm{X}$, is defined as $\mathrm{C}$

$$
C=B \Theta \mathrm{X}
$$




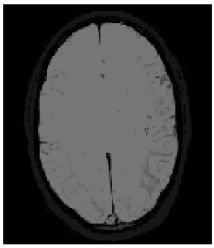

(a)

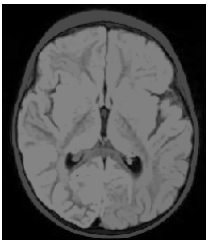

(b)
Fig -4: (a) and (b) Reconstructed images using input and eroded images.

\subsection{Dilation}

The dilation operator takes two pieces of data as inputs. The first is the image which is to be dilated. The second is a set of coordinate points known as a structuring element. It consists of a matrix of 0 's and 1's. The structural element can be a square, disk, and diamond shape. The dilation process is similar to the convolution process, that is, the structuring element is reflected and shifted from the left to right and from top to bottom, at each shift; the process will look for any overlapping similar pixels between the structuring element and that of the binary image. If there exists an overlapping then the pixels under the centre position of the structuring element will be turned to 1 or black.

Let us define $\mathrm{C}$ as the opening by reconstructed image and $\mathrm{X}$ as the structuring element. Dilated image defined $\mathrm{D}$

$$
D=C \oplus X=\{Z \mid[(\bar{X}) \mathrm{z} \cap \mathrm{X}] \sqsubseteq \mathrm{X}\}
$$

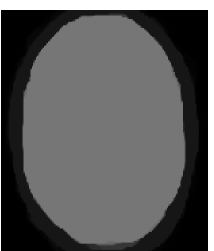

(a)

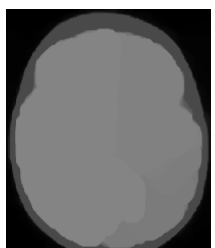

(b)
Fig -5: (a) and (b) Dilated images

\subsection{Opening Closing by Reconstruction}

Performing complement of opening by reconstruction image is termed as opening closing by reconstruction. The opening and closing by reconstruction of a image $\mathrm{D}$ is defined as $\mathrm{E}$

$$
\mathrm{E}=\bar{D}
$$

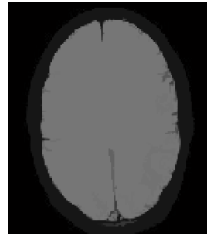

(a)

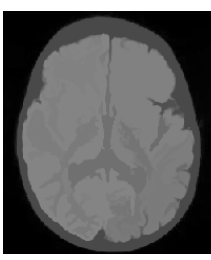

(b)
Fig- 6: (a) and (b) Reconstructed images

\subsection{Thresholding}

Convert the gray scale image into binary image by Thresholding. The output binary image $F$ has values of 1 (white) for all pixels in the input image with luminance greater than LEVEL and 0 (black) for all other pixels. LEVEL is based Global image threshold using Otsu's method. Binarized image consist 1 for brain tissues and 0 for non brain tissues. The binary image of a opening closing by reconstructed image $\mathrm{E}$ is defined as $\mathrm{F}$

$$
F= \begin{cases}0, & E<T \\ 1, & E \geq T\end{cases}
$$

Where $\mathrm{T}$ is the threshold value

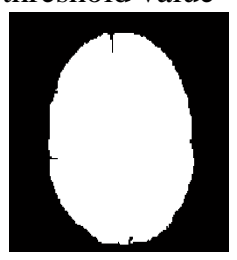

(a)

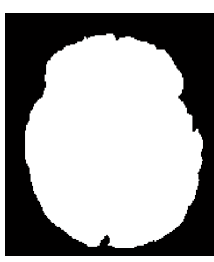

(b)
Fig -7: (a) and (b) Binarized image

\subsection{Final Output}

By setting the threshold condition with binarized image and input brain image, wherever the binarized image consist 1 place intensity level of input image and wherever the binarized image consist 0 place 0 .The output image consists only the brain tissues. The final output image defined as $\mathrm{G}$, binarized image as $\mathrm{F}$ and input image as $\mathrm{A}$

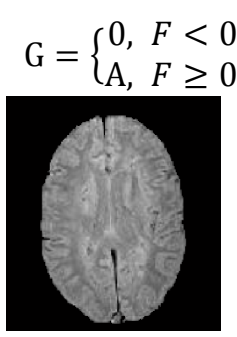

(a)
(6)

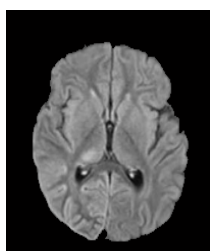

(b)
Fig -8: (a) and (b) Skull Stripped Image 


\section{EXPERIMENTAL RESULTS}

The proposed method was applied on the selected Axial PD and FLAIR oriented images. The results are shown in figure 12
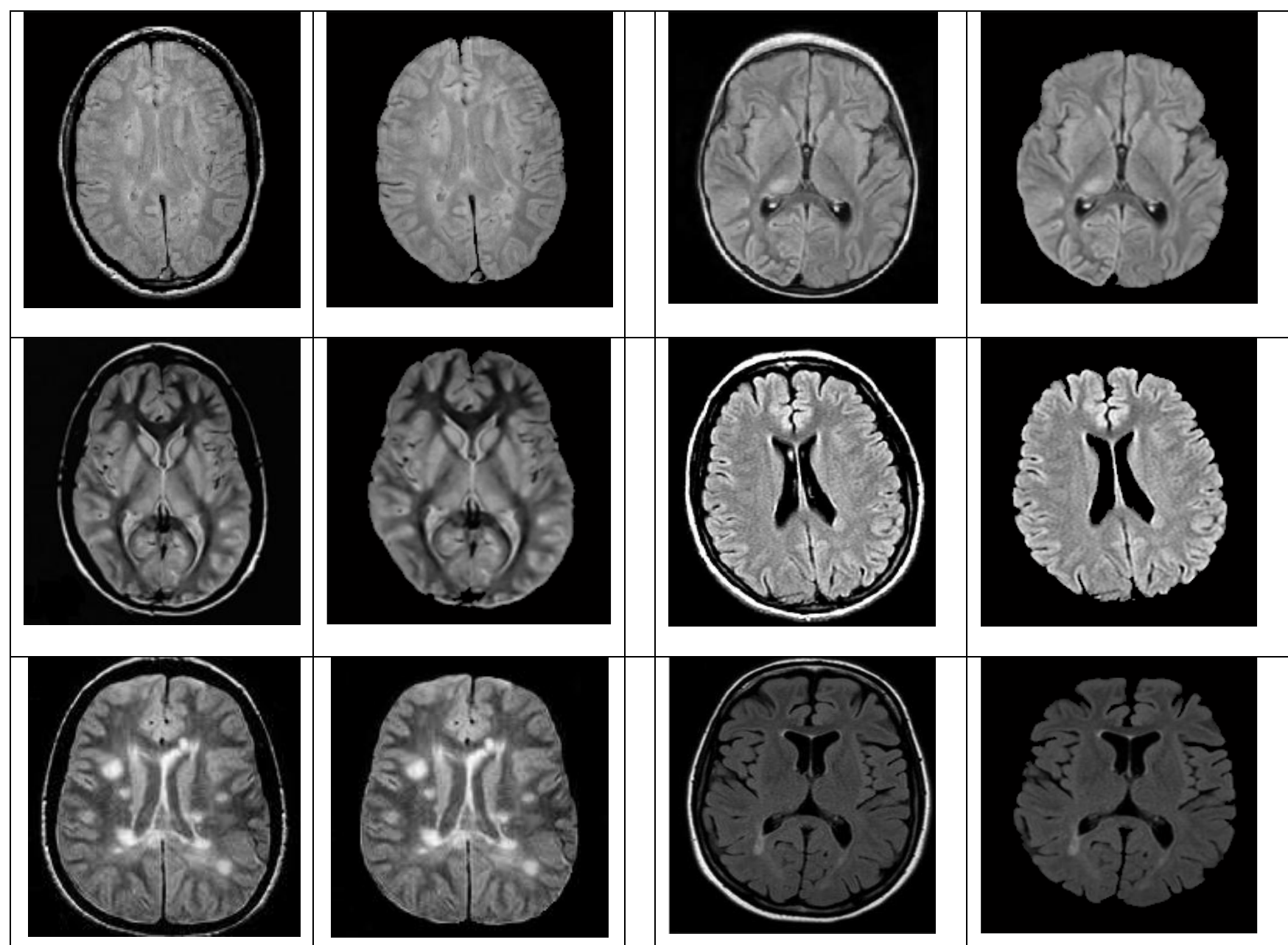

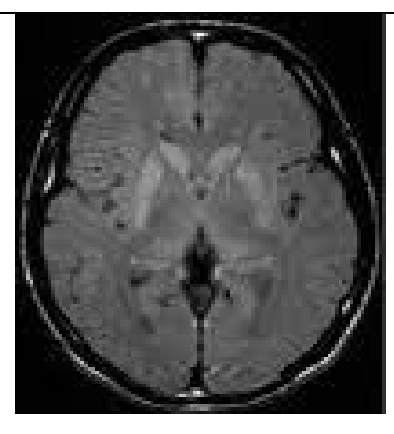

(a) Original MRI

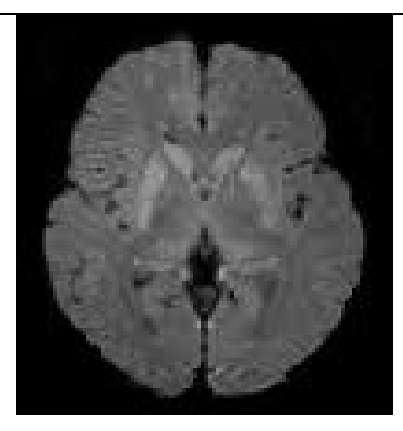

(b) Extracted Image

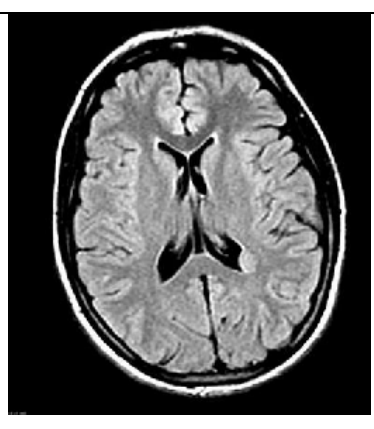

(a) Original MRI

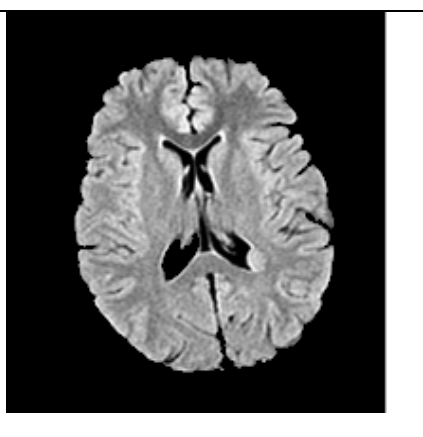

(b) Extracted Image

Fig -9: Brain portion extracted by the proposed method. Column 1 shows Original PD weighed MRI brain, Column 2 shows the extracted brain by proposed method Column 3 shows Original FLAIR weighted MRI brain, and Column 4 shows the extracted brain by the proposed method. 
The obtained results were compared with manually stripped images by finding $\mathrm{J}$ and $\mathrm{D}$ similarities,

\subsection{Jaccard similarity coefficient:}

The Jaccard similarity coefficient $\mathrm{J}$ is given as $\mathrm{J}=\operatorname{Vol}(\mathrm{A} \cap \mathrm{B}) / \operatorname{Vol}(\mathrm{A} \cup \mathrm{B})$, where $\mathrm{A}$ is the brain region of the manually stripped image and $B$ is the brain region of the skull stripped image obtained by the proposed method.

\subsection{Dice similarity coefficient:}

The Dice similarity coefficient $\mathrm{D}$ is given as $\mathrm{D}=2 \operatorname{Vol}(\mathrm{A} \cap \mathrm{B}) / \operatorname{Vol}(\mathrm{A}+\mathrm{B})=2 \mathrm{~J} /(1+\mathrm{J})$, where $\mathrm{A}$ is the brain region of the manually stripped image and $B$ is the brain region of the skull stripped image obtained by the proposed method.

Table -1: J and D similarities for Axial PD image

\begin{tabular}{|c|c|c|c|c|}
\hline $\begin{array}{c}\text { Data } \\
\text { set }\end{array}$ & $\begin{array}{c}\text { No of } \\
\text { Pixels } \\
\text { matched }\end{array}$ & $\begin{array}{c}\text { Total } \\
\text { No of } \\
\text { Pixels }\end{array}$ & J & D \\
\hline 1 & 37953 & 40000 & 0.9488 & 0.9737 \\
\hline 2 & 28735 & 40000 & 0.7184 & 0.8361 \\
\hline 3 & 35165 & 40000 & 0.8791 & 0.9356 \\
\hline 4 & 31553 & 40000 & 0.7888 & 0.8819 \\
\hline
\end{tabular}

Table -2: J and D similarities for Axial FLAIR image

\begin{tabular}{|c|c|c|c|c|}
\hline $\begin{array}{c}\text { Data } \\
\text { set }\end{array}$ & $\begin{array}{c}\text { No of } \\
\text { Pixels } \\
\text { matched }\end{array}$ & $\begin{array}{c}\text { Total } \\
\text { No of } \\
\text { Pixels }\end{array}$ & J & D \\
\hline 1 & 35242 & 40000 & 0.8811 & 0.9367 \\
\hline 2 & 36255 & 40000 & 0.9064 & 0.9509 \\
\hline 3 & 35398 & 40000 & 0.8850 & 0.9389 \\
\hline 4 & 36543 & 40000 & 0.9136 & 0.9548 \\
\hline
\end{tabular}

\section{DISCUSSION}

A total of 4 images were taken for axial PD and axial FLAIR images respectively. The J\&D similarity coefficients were found for the manually stripped and the images obtained by the proposed skull stripping method. The average values of $\mathbf{J}$ and $\mathrm{D}$ similarities are given in table 3.

\begin{tabular}{|c|c|c|}
\hline \multirow{2}{*}{ Brain } & \multicolumn{2}{|c|}{ Average } \\
\cline { 2 - 3 } Orientation & $\mathrm{J}$ & $\mathrm{D}$ \\
\hline Axial PD & 0.8337 & 0.9068 \\
\hline Axial FLAIR & 0.8965 & 0.9453 \\
\hline
\end{tabular}

Table 3: Average values of $\mathrm{J}$ and $\mathrm{D}$ similarities

It is found that the average $\mathbf{J}$ similarity value is greater than 0.8 where as the average value of D similarity is greater than 0.9 . Hence it is proved that the proposed method is efficient for skull stripping.

\section{CONCLUSION}

In this Paper a novel method to extract brain portion from Axial PD Weighted and Axial FLAIR weighted MRI images has been proposed. This method is able to detect the boundary separating brain and skull directly. The result shows that it is a reliable method for brain extraction. This method is also very simple.

\section{REFERENCES}

[1].Neha Tirpude, R.R.Welekar, "A study of brain magnetic resonance image segmentation techniques", International Journal of Advanced Research In computer and communication engineering, Vol.2, Issue 1, January 2013.

[2].D.Shattuck, S. Sandor-lealy, K.Schaper, D.Rottenberg and R.Leahy, "Magnetic resonance image tissue classification using a partial volime model", Neuroimage, 13, 856-876, 2001.

[3].T.Kapur, W.E.L Grimson, W.M.Wells and R.Kikinis, "Segmentation of brain tissue from Magnetic Resonance Images", Medical image analysis, Volume 1, No.2, pp 109-127, 1996.

[4].M.S Atkins and B.T.Mackiewich , "Fully automatic segmentation of the brain in MRI", IEEE transactions medical Imaging, 17(1),98-107, 1998.

[5].A.F.Goldszal, C.Davatzikos, D.L.Pham, M.X.H.Yan, R.N.Bryan and S.M.Resnick, "An image processing system for qualitative and quantitative volumetric analysis of brain images", journal comput.assisst. tomogr., 22(5):827-837,1998

[6].R.C.Gonzales amd R.E.Woods, Digital image processing, Second edition, Prentice Hall, 2002.

[7].Sajjad Mohsin, Sadaf Sajjad, Zeeshan Malik, and Abdul Hanan Abdullah, "Efficient way of skull stripping in MRI to detect brain tumor by applying morphological operations, after detection of false background",IJIET Vol.2, No.4, August 2012.

[8].K.Somasundaram, and R.Siva Shankar, "Skull stripping based on clustering and curve fitting with quadraric equations", ICMMSC, CCIS 283, PP 439-444. Springer Verlag Berling Heidelberg 2012.

[9].Orazio Gambini, Enrico Daidne, Maetteo Sciortino, Roberto Pirrone, "Auotmatic Skull Stripping in MRI based on Morphological Filtera and Fuzzy C-means Segmentation", $33^{\text {rd }}$ Annual International Conference of the IEEE EMBS, August 30, 2011.

[10].D.L.Pharm, J.L.Prince, "Adaptive Fuzzy segmentation of magnetic resonance images", IEEE Transactions on Medical Imaging, Vol 18(9), pp 737-752, 1999.

[11].Rosniza Roslan, Nursuriati Jamil and Rozi Mahmud, "Skull Stripping Magnetic Resonance Images Brain Images: Region growing versus Mathematical Morphology", International Journal of Computer Information Systems and Industrial Management Applications, Vol 3, pp 150-158, 2011. 
[12].R.Adams and L.Bischof, "Seeded region growing", IEEE Transactions on pattern Analysis and machine Intelligence. Vol 16(6), 641-646, 1994.

[13] .S.A.Hojjatoleslami and J.Kittler, "Region Growing: A new approach", IEEE Trans. Image Processing, Vol7, pp 1079-1084, 1998.

[14] .Jianping Fan, Guihua Zeng, Mathurin Body, MohandSaid Haci, Seeded region growing: An extensive and comparative study, Pattern Recognition Letters, Vol.26, pp. 1139-1156, 2005.

[15] .J.G. Park and C.Lee, "Skull stripping based on region growing for magnetic resonance brain images", Neuroimage, 47, 1394-1407, 2009.

[16] .F.Segonne, A.M.Dale, E. Busea, M.Glessner, D. Salat, H.K Hann and B.Fischl, "A hybrid approach to the skull stripping problem in MRI", Neuro Image 22 (2004), pp 1060-1075

[17] .H.K.Hann and H.O.Peitgen, "The skull stripping problem in MRI solved by Single watershed transform",Proc. Of Medical Image Computing and Computer Assissted Internention(MICCAI), LNCS, Vol 1935, pp 134-143(2000).

[18] .V.Grau, A.U.J.Mewes, M.Alcaniz, R.Kikinis , S.K.Warfield, "Improved watershed transform for medical image segmentation using prior information", IEEE Transactions on Medical Imaging, Vol 23(4), pp 447-458,2004.

[19] .A.H.Zhuang, D.J.Valentino and A.W.Toga, "Skull Stripping Magnetic Resnance Image Using A modelbased Level Sets", Neuro Image, Vol 32, pp79-92, 2006.

[20] .K.Somasundaram, T.kalaiselvi, "A comparative study of segmentation techniques used for MR Braim images", International Conference on Image processing, computer vision and pattern recognition, IPCv 2009, July 1316,2009, Las Vegas, Nevada, USA.

[21] .W.M.Wells, W.E.L.Grimson, R.Kikinis and F.A.Jolosz, "Adaptive segmentation on MRI Data", IEEE Trans. Medical Imaging, Vol 15, pp 429-443, 1996. 